\title{
Effect of Anti-tubercular Drugs and Impact of Glycemic Status in Patients with Tuberculosis and Concomitant Diabetic- Tuberculosis Patients
}

\author{
Nimmy Njattmkalayil John', Jane Mary George ${ }^{2}$, Narmadha Mukunda Poornachary ${ }^{2}$ \\ 'Department of Pharmacy Practice, Krupanidhi College of Pharmacy, Bangalore, Karnataka, INDIA. \\ ${ }^{2}$ Department of Pharmacy Practice, Swamy Vivekandha College of Pharmacy, Erode, Tamil Nadu, INDIA
}

\begin{abstract}
India is a key nation which is endemic and epidemic to tuberculosis and diabetes. An association between diabetes and TB is biological plausible, where diabetes impairs the immune system and making it harder for the body to fight against infection. Methodology: Patients on DOTS therapy in continuation phase of anti-TB therapy, Patients with TB and Diabetes and Patients with TB alone were included in the study. Blood samples were collected and Fasting Blood Glucose (FBG), Post Prandial Glucose (PPG) and Glycated Hemoglobin (HbA1c) were measured to understand the glycemic status and its impact on TB treatment outcomes. Results: Our study confirms the fact that type II diabetes is a strong risk factor for tuberculosis and is associated with a slower response to TB treatment and a higher mortality rate. Incidence of TB is greatest among those with conditions impairing immunity such as DM. Diabetes impairs the immune system, making it harder for the body to fight against infection.
\end{abstract}

Keywords: Tuberculosis, Glycemic status, Lung field, Diabetes Mellitus, Glycated Hemoglobin.

\section{INTRODUCTION}

The global burden of disease from diabetes and tuberculosis is immense. ${ }^{1}$ India is a key nation which is endemic and epidemic to tuberculosis and diabetes. ${ }^{1,2}$ Tuberculosis is a major public health problem where India accounts for one fifth of the global burden of TB incident cases. Each year nearly 2 million people in India develop TB, of which around 0.87 million are infectious cases. It is estimated that annually around 3,30,000 Indians die due to TB. ${ }^{3}$

An association between diabetes and TB is biological plausible, for diabetes impairs the immune system, making it harder for the body to fight against infection. ${ }^{4}$ Clinicians have observed an association between $\mathrm{DM}$ and TB, although they were often unable to determine whether DM caused by TB or whether TB led to the clinical manifestations of DM. Multiple rigorous epidemiological studies, have demonstrated that DM is indeed positively associated with TB.
A causal link between DM and TB does not bode well for the future, as the global burden of DM is expected to rise from an estimated 180 million prevalent cases currently to a predicted 366 million by 2030. Experts have raised concerns about the merging epidemics of DM and TB. Prevalence of tuberculosis among diabetic is 2.5 times higher than in the nondiabetic population, and a higher rate of lower lung field involvement seen in diabetic patients. ${ }^{5}$ In $85 \%$ of the patients, tuberculosis had developed after the onset of diabetes. The association is more common among those who are above 40 years of age and males appear to be at a greater risk compared with females. Diabetes may also complicate the management of tuberculosis. ${ }^{6}$

TB is more common in patients with diabetes, especially in those with poor glucose control. Diabetes has been associated with increased risk of TB treat-
DOI: 10.5530/ijopp.10.2.25

Address for correspondence:

Nimmy N John,

Assistant Professor, Department of Pharmacy Practice Krupanidhi College of Pharmacy, Bangalore, Karnataka, INDIA

Phone no: +91 7349550506

E-mail: nimmyasish@gmail.com 
ment failure or relapse, and diminished 2 month and 6 month culture conversion rates.

Hence this study was aimed to find out the effect of anti-TB drugs in patients with TB alone and TB with Diabetes groups.

AIM: To find out the effect of anti-TB drugs in patients with TB alone and TB with Diabetes groups.

\section{MATERIAL AND METHODS}

The protocol was accepted by Institutional Ethics Committee. It was a Prospective case control study conducted at a 1000 bedded tertiary care hospital at Tamil Nadu. Patients on DOTS therapy in continuation phase of anti-TB therapy. Patients with $\mathrm{TB}$ and Diabetes and Patients with TB alone were included in the study whereas patients who are HIV positive, pregnant and lactating women, abnormal renal functions and abnormal liver functions were excluded from our study. Patients underwent tests for Blood glucose and lipid profile. Blood samples were collected and Fasting Blood Glucose (FBG), Post Prandial Glucose (PPG) and Glycated Hemoglobin (HbA1c) were measured to understand the glycemic status and its impact on TB treatment outcomes. Similarly, Total Cholesterol, Triglycerides, HDL and LDL were also measured to find whether any variation in these among the test and control groups.

\section{RESULT}

In the study, no statistically significant difference was found in exposure of age, vital studies and lipid profile, while the statistically significant increase was seen in FBG, PPG and HbA1c in diabetic patients with TB than in patients with TB and without diabetes (Table 1).

The Table 2 explains the difference in glycemic status among the test and control groups. The TBDM group had shown an extremely significant increase in FBG (P value 0.0231)

From the Table 3, it is observed a slight variation in lipid profile among the TBDM and TB groups. Total cholesterol was slightly higher in TB group where the triglycerides, HDL and LDL values were greater in TBDM group. But no significant changes were found.

Considering the category of treatment taken, the patients with diabetes along with tuberculosis were found with a higher fasting and post prandial glucose levels in Cat. II patients than in Cat. I patients, where, HbA1c (glycated hemoglobin) values were
Table 1: Effect of anti-TB drugs in patients with TB alone and TB with Diabetes group.

$\begin{array}{cccc} & \begin{array}{c}\text { TBDM } \\ \text { group }\end{array} & \begin{array}{c}\text { TB } \\ \text { group }\end{array} & \text { P value } \\ \text { Age (28-60) } & 49 & 46 & 0.2742 \\ \text { Karnofsky Performance } & 72.5 & 75 & 0.3093 \\ \text { Status Scale (60-90) } & & & \\ \text { Temperature (F) } & 98.6 & 98.6 & 0.1426 \\ \text { Pulse rate (bts/min) } & 74 & 74 & 0.3906 \\ \text { Systolic BP (mmHg) } & 123 & 125 & 0.2696 \\ \text { Diastolic BP (mmHg) } & 84 & 80 & 0.0447 \\ \text { FBG (mg/dl) } & 179 & 72 & 0.0231 \\ \text { PPG (mg/dl) } & 318 & 115 & 0.0009 \\ \text { HbA1c (\%) } & 7.7 & 5.5 & <0.0001 \\ \text { Total Cholesterol (mg/dl) } & 176 & 182 & 0.2616 \\ \text { Triglycerides (mg/dl) } & 164 & 158 & 0.1610 \\ \text { HDL }(\mathrm{mg} / \mathrm{dl}) & 47 & 44 & 0.2414 \\ \text { LDL }(\mathrm{mg} / \mathrm{dl}) & 109 & 104 & 0.2906\end{array}$

Table 2: Comparison of Glycemic status among the test (TBDM) and control (TB) group

$$
\text { TBDM Mean } \pm \text { SD TB Mean } \pm \text { SD (SEM) }
$$$$
\text { (SEM) }
$$

$\mathrm{FBG}(\mathrm{mg} / \mathrm{dl})$

$179.38 \pm 138.03$

$(48.80)$

PPG $(\mathrm{mg} / \mathrm{dl}) \quad 317.75 \pm 148.25$

(52.415)

$71.750 \pm 17.393$

(6.149)

$115.13 \pm 21.263$

(7.518)

$\mathrm{HbA} 1 \mathrm{c}(\%) \quad 7.738 \pm 0.5397$

(0.1908)

$5.475 \pm 0.2866$

(0.1013)

(P value 0.0009) and $\mathrm{HbA} 1 \mathrm{C}(\mathrm{P}$ value $<0.0001$ ) levels than the TB alone group

$\begin{array}{ccc}\begin{array}{l}\text { Table 3: Comparison of Lipid profile among TBDM } \\ \text { and TB groups }\end{array} & & \\ & \text { TBDM Mean } \pm \text { SD } & \text { TB Mean } \pm \text { SD } \\ \text { (SEM) } & \text { (SEM) } \\ \text { Total Cholesterol } & 175.88 \pm 23.333 & 181.88 \pm 11.269 \\ \text { (mg/dl) } & (8.249) & (3.984) \\ \text { Triglycerides (mg/dl) } & 164 \pm 8.106 & 157.75 \pm 15.192 \\ & (2.866) & (5.371) \\ \text { HDL (mg/dl) } & 46.750 \pm 8.812 & 43.500 \pm 9.212 \\ & (3.115) & (3.257) \\ \text { LDL (mg/dl) } & 108.75 \pm 16.202 & 103.75 \pm 19.092 \\ & (5.728) & (6.750)\end{array}$

found higher in Cat. I patients than in Cat. II patients. There was not much marked inter-individual variability was observed in patients with tuberculosis and without diabetes, based on category of treatment taken. 
Table 4: Comparison of Glycemic status of patients in TBDM and TB group

\begin{tabular}{ccccc} 
& \multicolumn{2}{c}{ TBDM } & \multicolumn{2}{c}{ TB } \\
& \multicolumn{2}{c}{ Median(Range) } & \multicolumn{2}{c}{ Median(Range) } \\
& Cat. I & Cat .ll & Cat. I & Cat. II \\
FBG (mg/dl) & 124 & 323 & 67 & 65 \\
& $(57-158)$ & $(107-455)$ & $(57-105)$ & $(57-73)$ \\
& \multicolumn{4}{c}{} \\
PPG (mg/dl) & 280 & 448 & 121 & 101.5 \\
& $(166-338)$ & $(218-611)$ & $(105-136)$ & $(71-132)$ \\
HbA1c (\%) & 8.1 & 7.4 & 5.65 & 5.3 \\
& $(6.9-8.5)$ & $(7.1-7.8)$ & $(5.1-5.9)$ & $(5.3-5.3)$ \\
& & & &
\end{tabular}

\section{DISCUSSION}

Our study confirms the fact that type II diabetes is a strong risk factor for tuberculosis and is associated with a slower response to $\mathrm{TB}$ treatment and a higher mortality rate. ${ }^{7,99}$ Incidence of TB is greatest among those with conditions impairing immunity such as DM. Diabetes impairs the immune system, making it harder for the body to fight against infection. ${ }^{1,10}$

Earlier studies shows that tuberculosis and diabetes mostly affects males than females, and also the patients with DM above 40 years are vulnerable to TB. ${ }^{8,9}$ In our study too males were found to be affected more than females and they were of age above 30 year old with DM along with TB.

In our study it was painful to note that all the patients recruited in the study were alcoholic and smokers at the onset of TB. Since they were advised to stop alcohol and smoking in order to make treatment effective they had stopped. The tobacco smoke and alcohol abuse are major risk factors which reduces the effectiveness of $\mathrm{TB}$ treatment and increases prevalence of TB. $3,7,9$

Among the patients with DM in anti-TB therapy around $90 \%$ cases were pulmonary tuberculosis with $70 \%$ sputum positive cases. The studies done earlier had shown a high percentage of sputum positivity and a higher rate of pulmonary TB than extra pulmonary tuberculosis in diabetic patients. $1,6,9,10$ It is because glucose stimulates the mycobacterial growth ${ }^{5}$ and uncontrolled DM is responsible for poor clinical response to anti-TB therap $\mathrm{y}^{6}$ which also increases the susceptibility to mycobacterial growth. ${ }^{1,8}$

The patients with diabetes along with TB will be having a lower health performance as these disease affects the host defenses and immunity of body, and thereby unable to resist against infections and other comorbidities. ${ }^{1,8}$

\section{CONCLUSION}

India is a developing country which is endemic and epidemic to tuberculosis and diabetes. Our study also had shown the need to improve the care of patients with concomitant DM and TB. Enhanced medical vigilance, especially in patients with diabetes is required in tuberculosis population. It seems to be rational to screen for $\mathrm{DM}$ in patients with $\mathrm{TB}$ and vice versa, as these may have implications for control and treatment of both diseases. Therapeutic monitoring of anti-TB drugs along with regular monitoring of glucose levels using insulin can be recommended in patients with diabetes and tuberculosis.

Our study also recommends TB control program should consider targeting patients with diabetes for interventions such as active case finding and treatment of latent $\mathrm{TB}$ and conversely that effort to diagnose detect and treat diabetes may have a beneficial impact on TB control.

\section{ACKNOWLEDGEMENT}

Authors acknowledging the co-operation and support provided by the medical team of Government Hospital, Namakkal.

\section{CONFLICT OF INTEREST}

\section{Nil}

\section{ABBREVIATIONS USED}

TB: tuberculosis; DM: Diabetes mellitus; TBDM: tuberculosis and DIabetes mellitus; DOTS: directly observed treatment short course; HIV: human immunodeficiency virus; FBG: fasting blood glucose; PPG: post prandial glucose; HDL: High density lipoprotein; LDL: low density lipoprotein; $\mathrm{Hb}$ A1c: Glycated hemoglobin.

\section{REFERENCES}

1. Jeon CY, Murray MB. Diabetes Mellitus Increases the Risk of Active Tuberculosis: A Systematic Review of 13 Observational Studies. www. plosmedicine.org. 2008;5(7):e152.

2. Balasubramanian $\mathrm{R}$, et al. Evaluation of an intermittent six-month regimen in new pulmonary tuberculosis patients with diabetes mellitus. Indian J Tuberc. 2007;54(4):168-76

3. Anthony D. Harries et al, Defining the research agenda to reduce the joint burden of disease from Diabetes mellitus and Tuberculosis. Tropical Medicine and International Health. 2010;15(6):659-63.

4. Fraser A, Paul M, Attamna A, Leibovici L. Drugs for preventing tuberculosis in people at risk of multiple drug-resistant pulmonary tuberculosis. The Cochrane Library. 2009:3.

5. Diabetes May Threaten TB Control. www.india-server.com/.../diabetes-maythreaten-tb-control-2554.html. 
6. Lu FB, Basoglu OK, Abdullah GC, Ates SM. Pulmonary Tuberculosis in Patients with Diabetes mellitus. Respiration. 2001;68:595-600.

7. Leung CC, et al. Diabetic Control and Risk of Tuberculosis: A Cohort Study. Am J Epidemiol. 2008;167(12):1486-94.

8. Kotokey RK, Bhattacharya DN, Das P, Azad AK, De A. Study of efficacy of dots in pulmonary tuberculosis patients with associated diabetes. Lung India. 2007;24(2):58-60.

9. Jain MK, Baghel PK, Agrawal R. Study of Impaired Glucose Tolerance in Pulmonary Tuberculosis. Indian Journal of Community Medicine. 2006;31(3)137-9.
10. Nijland HM, et al. Exposure to Rifampicin Is Strongly Reduced in Patients with Tuberculosis and Type 2 Diabetes. Clinical Infectious Diseases. 2006;43(7):848-54.

11. Nissapatorn V, et al. Tuberculosis In Diabetic Patients: A Clinical Perspective. Southeast Asian J Trop Med Public Health. 2005;36(Suppl 4)213-20.

12. Kotokey RK, Bhattacharya DN, Das P, Azad AK, De A. Study of efficacy of dots in pulmonary tuberculosis patients with associated diabetes. Lung India 2007;24(2):58-60. 